\title{
Fragment-Specific Fixation in Distal Radius Fractures
}

\author{
Bryan A. Hozack ${ }^{1} \cdot$ Rick J. Tosti ${ }^{2}$ \\ Published online: 5 March 2019 \\ (C) Springer Science+Business Media, LLC, part of Springer Nature 2019
}

\begin{abstract}
Purpose of Review Fragment-specific fixation can be a useful tool in treating distal radius fractures. In order to best utilize these techniques, surgeons require an understanding of the normal anatomy, fracture patterns, approaches, and fixation strategies.

Recent Findings Fragment-specific fixation may be appropriate for certain fracture patterns particularly when monoblock plating techniques are not sufficient.

Summary Radial styloid, volar rim, dorsal wall, dorsal-ulnar corner, and impacted intraarticular fragments may be secured with implants designed specifically for each individual fragment. Although more technically demanding, advantages include accurate articular reconstruction, minimal hardware irritation, and reliable functional outcomes.
\end{abstract}

Keywords Distal radius fractures $\cdot$ Intraarticular fractures $\cdot$ Fragment-specific fixation

\section{Introduction}

Distal radius and ulna fractures are the most common fracture in adults representing $2.5 \%$ of all emergency room visits and $44 \%$ of all fractures $[1,2]$. Nonoperative management can be successful for stable fractures. However, displaced or unstable fractures are usually indicated for operative treatment in order to restore alignment, function, and prevent premature arthrosis (Table 1).

Surgeons may choose from several operative options depending on the fracture type. Fragment-specific fixation is usually indicated in cases in which stabilization of specific articular segments is required. Additionally, fragmentspecific fixation may augment monoblock or spanning plates especially to reconstruct articular congruity. Although fracture morphology has been described in a variety of classification systems, we find the fragment-

This article is part of the Topical Collection on Distal Radius and Wrist Fractures

Bryan A. Hozack

bhozack@gmail.com

1 Rothman Institute at Thomas Jefferson University Hospital, 925 Chestnut Street, 5th Floor, Philadelphia, PA 19107, USA

2 Orthopaedic Surgery, Hand to Shoulder Center, Thomas Jefferson University, 834 Chestnut Street Suite G114, Philadelphia, PA 19107, USA specific classification system described by Medoff most applicable to these techniques [3] (Fig. 1). However, we intend discuss several classification systems as they relate to the common fragments.

The following article will discuss the anatomy of the distal radius, the pathomechanics and features of common articular fragments, surgical approaches, techniques to restore small articular segments, and the outcomes to be expected.

\section{Radial Styloid}

\section{Anatomy}

The articular surface of the radial styloid is concave and is the apex of the triangle that makes up the distal radiocarpal articulation [4]. The main ligamentous attachment of the volarradial styloid includes the radioscaphocapitate (RSC) ligament and a portion of the long radiolunate (LRL) ligament. The RSC ligament constrains carpal pronation and provides restraint to ulnar translation of the carpus [5]. The LRL ligament moderately constrains carpal pronation. Dorsally, the radiotriquetral ligament provides stability in supination [6].

The superficial radial nerve lies in the subcutaneous fat proximal to the radial styloid, giving off a palmar branch before passing over the first dorsal compartment [7]. The cephalic vein lies radial and volar to the dorsal trunk of the 
Table 1 Radiographic parameters of fragments requiring operative reduction
Radial shortening $>5 \mathrm{~mm}$

Dorsal tilt $>10^{\circ}$ or volar tilt $>20^{\circ}$

Intraarticular step-off $>2 \mathrm{~mm}$

Radial inclination $>15^{\circ}$

superficial radial nerve, close to the radial styloid. The first dorsal compartment lies in a trough formed by fibro-osseous septae on the volar-radial and dorsal-radial aspect of the styloid [8]. The second dorsal compartment lies adjacent to the first, diverging at the mid to distal radial styloid toward the metacarpal bases [8]. The radial artery runs deep to the first dorsal compartment, distal to the styloid [9]. The brachioradialis has a broad, flat tendon with a footprint averaging $95.3 \mathrm{~mm}^{2}$, with a length of $15 \mathrm{~mm}$ and a width of $11 \mathrm{~mm}$ [10]. The distal point of the insertion is $17 \mathrm{~mm}$ proximal to the tip of the styloid and the proximal portion inserts $32 \mathrm{~mm}$ from the tip [10].

\section{Types of Fractures}

Fractures of the radial styloid usually occur due to compression or shear force of the scaphoid against the radius, but they occasionally result from an avulsion force as severe ulnar deviation and supination create tension along the RSC ligament, which may fail at its attachment to the styloid. Styloid fractures may be a component of distal radius fractures, carpal dislocations, and radiocarpal dislocations. Isolated radial styloid fractures were originally described in chauffer's who suffered shear-type injuries from the kickback of a crank on older cars [11]. Melone also described styloid fractures as they

related to a compression force. He noted 4 distinct fragments that commonly formed in distal radius fractures: radial shaft, dorsal medial fragment, palmar medial fragment, and radial styloid [11]. The AO/ASIF classification provides the most comprehensive classification of radial styloid fractures: B1.1 represents a simple fracture of the radial styloid, B1.2 represents a comminuted radial styloid fracture, B2.2 refers to distal radius fractures with dorsal subluxation and radial styloid involvement, and B2.3 refers to a radiocarpal dislocation with avulsion of the radial styloid by way of the radioscaphocapitate ligament [12]. Lastly, the Fernandez classification system refers to the mechanism of the distal radius fractures: bending fractures (type 1), shearing fractures of the joint surface (type 2), compression fractures (type 3), avulsion fractures (type 4), and combined fractures (type 5). The radial styloid fractures can be shearing (type 2) or avulsion (type 4) [13].

\section{Radial Approach}

The radial approach is along the radial aspect of the wrist directly over the radial styloid. The incision can be longitudinal or oblique. The radial sensory nerve should be identified in the subcutaneous tissue and retracted. The first dorsal compartment will be running on the volar aspect of the radial styloid, and the second dorsal compartment will be dorsal. The brachioradialis inserts onto the styloid deep to the first compartment. For exposure, the first compartment's tendon sheath can be released and the styloid can be exposed through the compartment floor between the abductor pollicis longus and the extensor
Fig. 1 Fragment-specific classification system described by Medoff

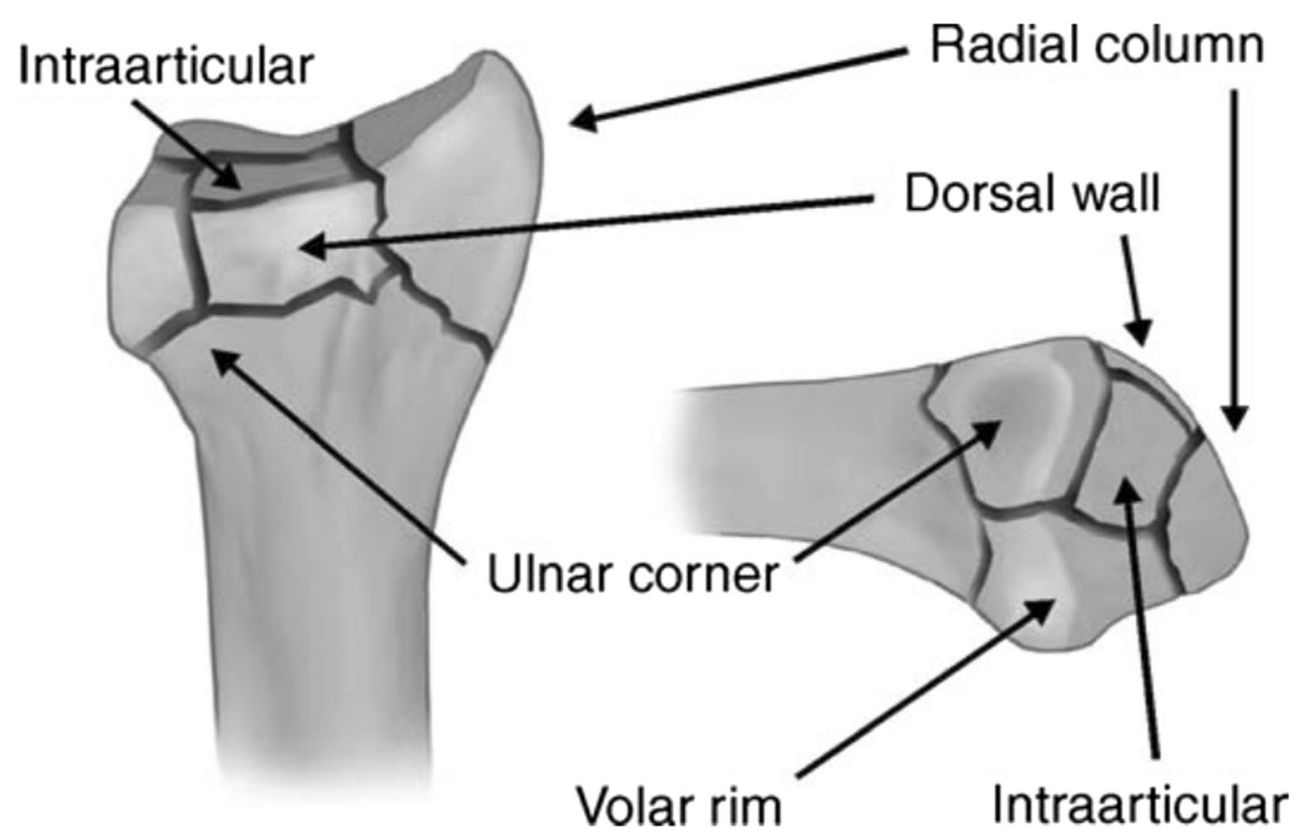


pollicis brevis. Additionally, the brachioradialis can be released proximally for better exposure and to alleviate the deforming force of the muscle $[10,14]$.

\section{Types of Implants}

\section{Plate}

This plate is ideal for radial styloid fractures with metaphyseal comminution, but can also be used in conjunction with other types of plating. After reduction of the fracture and placement of the plate over the radial styloid, the first screw is placed in the oblong slot to secure the plate to the radius. The distal locking and nonlocking screws can then be placed [15•].

\section{Pin Plate}

After reduction is confirmed with fluoroscopy, a K-wire is inserted into the radial styloid. The pin plate is placed over this $\mathrm{K}$-wire and secured to the radius with one of two screws proximally. An additional K-wire can be placed as well. Both $\mathrm{K}$-wires are backed out, bent, and impacted through the pin hole in the plate. Additional screws can be placed proximally for increased stability.

\section{Hook Plates}

Hook plates can be used for fixation of small, distal fragments that do not lend themselves to screw fixation. The hook is placed through the fragment, and the screw is placed proximally once reduction is obtained.

\section{Screws}

Simple fractures of the radial styloid can be reduced with a Kwire and secured with screws. The surgeon may select from headed, headless, cannulated, or solid screws to hold fixation (Fig. 2).

\section{Tension Band}

To counteract the wrist extension and ulnar deviation mechanism of certain radiocarpal dislocations, small radial styloid avulsion fractures can be secured with a tension-band construct to counteract the tensile forces [16].

\section{Outcomes}

The most common complication following radial styloidspecific fixation is transient radial sensory nerve neuropraxia [17••]. Patients occasionally require implant removal due to hardware irritation [18]. Radial styloid plates have also been used to assist in fracture reduction prior to volar plating. These

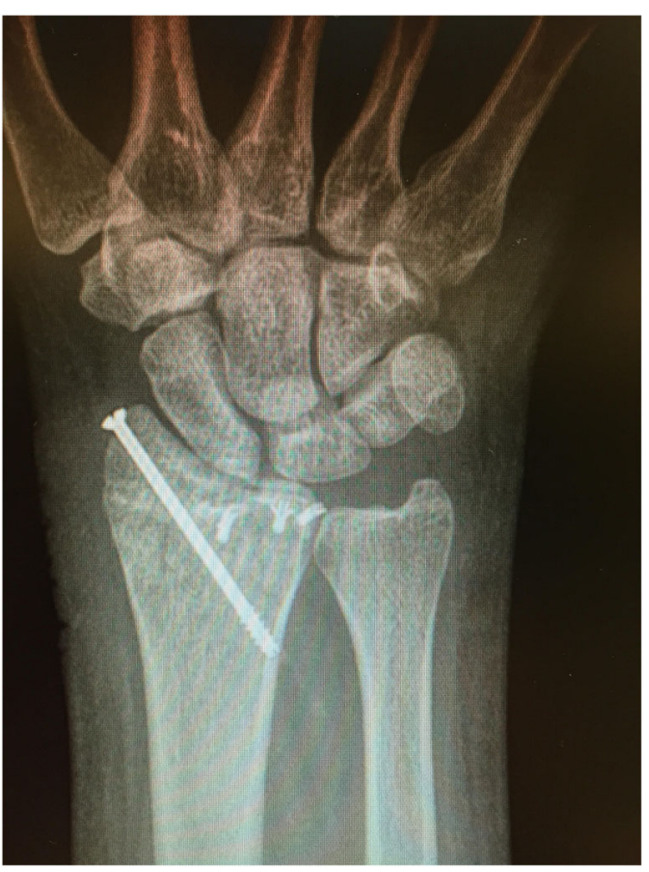

Fig. 2 A radiocarpal fracture dislocation treated with screw fixation of the styloid and bone anchors to repair the extrinsic ligaments

plates can be either removed at the end of the procedure or left in place. In one study, $36 \%$ of patients elected for implant removal at an average of 7 months following surgery [19], although in the authors' experience, this figure seems higher.

\section{Volar Rim}

\section{Anatomy}

The volar surface of the distal radius is relatively flat until the very distal margin, which slopes volarly to form a ridge from which the volar radiocarpal ligaments (short and long radiolunate ligaments) originate. When viewed in the axial plane in cross section, the distal cortical margin of the radius slopes volarly from the radial to ulnar aspect [20]. This corner of the radius imparts "critical" stabilization to the carpus and can be a common source of failure if not properly secured [11].

\section{Types of Fractures}

Volar rim fragments are usually formed from a shear force or by compression of the lunate against the radius. Occasionally, an avulsion fracture may pull small fragments from their ligamentous origins at the volar rim as well. The Fernandez classification type II would include volar shear fractures and type III would include compression fractures. In the AO classification system, B3.3 fractures involve a volar rim fragment typically resulting from a shear force. There can be volar rim 
fragments in $\mathrm{C} 1, \mathrm{C} 2$, or $\mathrm{C} 3$ fractures, which are typically more compression fractures.

\section{Volar Approach}

The volar extensile approach incorporates a carpal tunnel release and allows direct visualization and fracture reduction of the volar-ulnar corner of the distal radius [14, 21, 22]. The incision should be placed between the palmaris longus and flexor carpi ulnaris tendons. The incision is extended obliquely across the wrist to include a carpal tunnel release incision. The fascia is released between the flexor carpi ulnaris and the palmaris longus. The interval should be developed between the ulnar neurovascular bundle underneath the flexor carpi ulnaris ulnarly and the flexor digitorum superficialis/ profundus tendons radially. Moving distally, the ulnar neurovascular structures move ulnarly toward Guyon's canal and the finger flexor tendons can be retracted radially to expose the volar-ulnar corner of the distal radius and the floor of the carpal tunnel [14].

\section{Types of Implants}

\section{Buttress Pin Plate}

Once a $\mathrm{K}$-wire is placed to hold the reduction of the fracture fragment, a pin plate can be slid over the wire. A screw is placed proximally in the shaft to hold the plate reduced. The wire is then backed out, bent, and impacted into an adjacent hole. An additional K-wire can be placed distally, and screws can be placed proximally [23] (Fig. 3).

\section{Tension Wire}

For small, very distal fragments, a wire can be placed through the volar ligamentous attachments on the fracture fragment and looped in a figure-of-eight through two small bone tunnels made in the metaphysis. The tunnels can be made with a 2-mm drill and widened with a curette as necessary. Care should be taken not to fracture the bone bridge between the tunnels. The wire enters in one tunnel and exits the other before being twisted. This technique minimizes prominent hardware that becomes a major issue with very distal fracture fixation constructs [24] (Fig. 4).

\section{Hook Plate}

If the fragment is too small to capture with a standard volar plate or if the fracture is distal to the watershed line, a hook plate can be applied alternatively as either a separate construct or an extension of a volar locking plate. Once the volar plate is placed, fragment is predrilled to accommodate the hook plate, and the hook plate is fixed to the volar plate [25••].

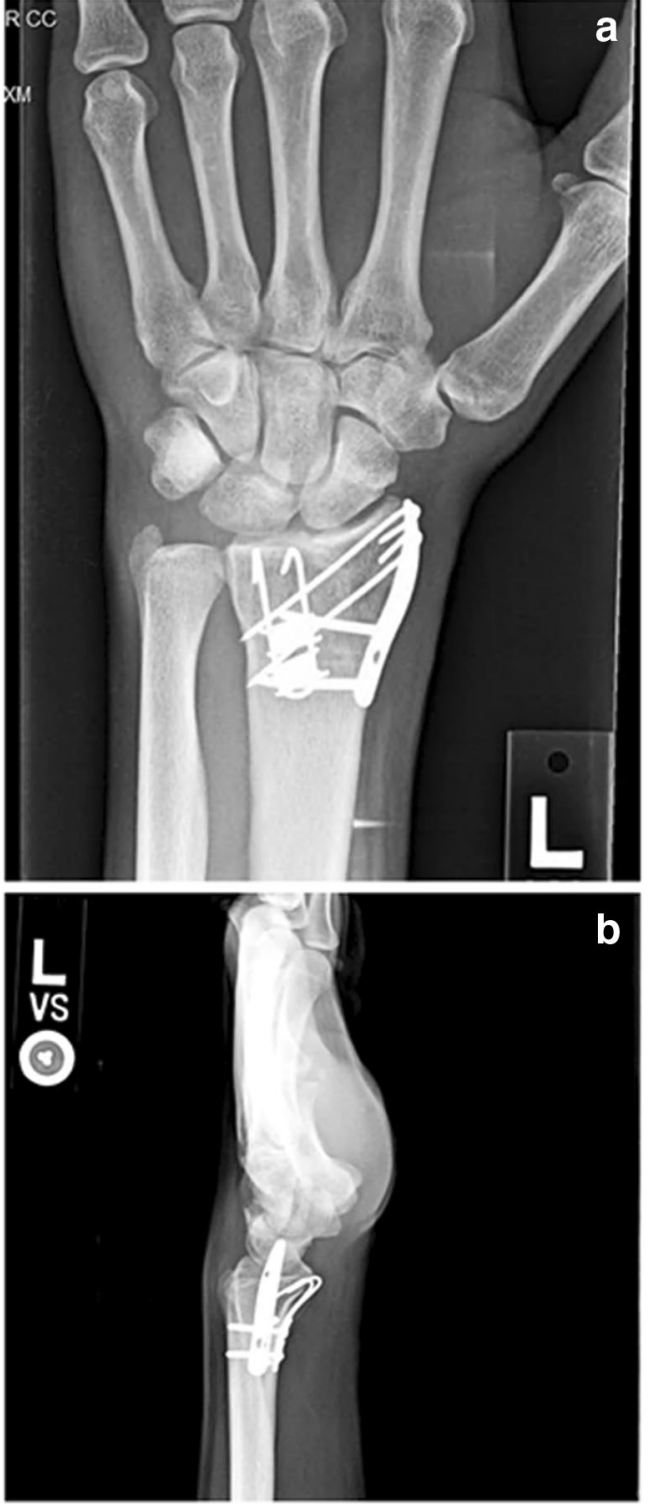

Fig. 3 a, b A radiocarpal fracture dislocation which involved the radial styloid and volar rim secured with fragment-specific fixation

\section{Outcome}

Volar lunate shear fractures are at risk of losing reduction and the hardware can be prominent, leading to tendon irritation requiring hardware removal. The carpus may translate volarly when the fragment is not properly reinforced [3]. Up to $13 \%$ of lunate facet fractures can lose reduction when a standard volar plate is used [26]. If a volar plate is used, it may need to be placed very distal in order to capture the fragment. As a result, hardware irritation is common in this location and may be present in up to $41 \%$ of fractures [27]. In another study, five out of 15 volar hook plates needed to be removed due to symptomatic hardware [25••]. Wire loop fixation had good results without any complications in a small series of distal lunate facet fractures [24]. 


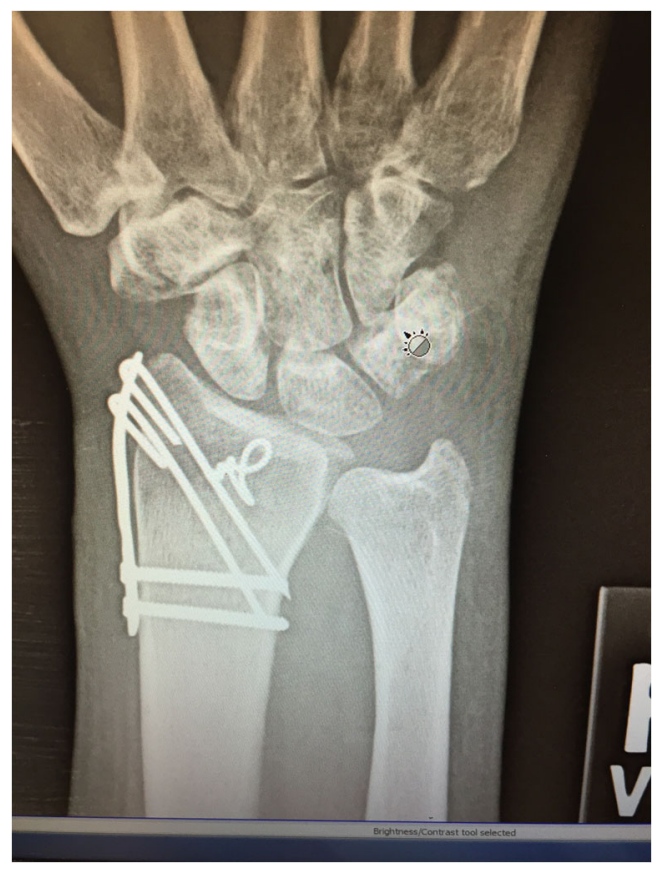

Fig. 4 A tension wire was used to secure a thin volar rim fragment

\section{Dorsal-Ulnar Corner}

\section{Anatomy}

The dorsal-ulnar corner of the radius includes the dorsal corner of the distal radioulnar joint. The dorsal-ulnar corner constitutes the bony rim of the sigmoid notch and serves as the origin of the dorsal radioulnar ligaments [9]. The triangular fibrocartilage complex arises at the ulnar aspect of the lunate facet of the distal radius and extends onto the base of the ulnar styloid process, functioning as an important stabilizer of the distal radioulnar joint. Its volar and dorsal margins are thickened, blending into the dorsal and volar radioulnar ligaments [28]. Rotation of the radius about the ulna is accompanied by translation such that in supination, the ulnar head displaces volarly in the notch, while in pronation, it moves dorsally. Displacement of the ulnar corner fragment in relation to the ulnar head can result in abnormalities of the distal radioulnar joint [3].

\section{Types of Fractures}

The dorsal-ulnar corner fragment may result from stresses of the lunate against the radius or the ulnar head against the sigmoid notch. It may be associated with incongruity of the distal radioulnar joint (DRUJ). These are generally noted in higher energy injuries with comminuted fractures. Medoff described the fragment in his classification system as did Melone in type III, IV, or V fracture patterns. The AO classification noted involvement in type B1.3, B2.3, C1.1, and C1.2 and all
C3 fracture patterns. In the Fernandez classification system, the dorsal-ulnar corner can be involved in type III and V fractures.

\section{Dorsal Approach}

The dorsal approach begins by identifying Lister's tubercle and making a longitudinal incision in line with the third metacarpal just ulnar to Lister's tubercle. Dissection proceeds down to the extensor retinaculum and full thickness skin flaps should be raised, which will protect the branches of the radial sensory and dorsal-ulnar sensory nerves and any dorsal veins. The extensor pollicis longus can be identified ulnar to Lister's tubercle. An incision through the extensor retinaculum and the roof of the extensor pollicis longus is made, releasing the extensor pollicis longus, which is retracted radially. The second and fourth compartments are raised under the periosteum in opposite directions to expose the dorsal surface of the radius [14]. The posterior interosseous nerve lies proximally within the floor of the fourth dorsal compartment. It provides sensation and proprioception to the dorsal wrist capsule at this level [29]. It may be excised if arthrosis or pain is anticipated. If visualization of the radiocarpal joint is required, a capsulotomy can be made in this interval in order to treat concomitant intracarpal injuries.

\section{Dorsal-Ulnar Approach}

The dorsal-ulnar approach provides direct visualization of the dorsal-ulnar corner of the distal radius [14]. The distal radioulnar joint is palpated and a longitudinal incision is placed above it. The fifth dorsal compartment can be identified by visualizing the extensor digiti minimi exiting distally. This compartment can be released and the distal radioulnar joint is deep to its floor. Care should be taken to preserve the distal radioulnar ligaments, as excessive stripping may lead to instability of the DRUJ.

\section{Type of Implants}

\section{Dorsal Pin Plate}

Similar to other pin plates, a $\mathrm{K}$-wire is placed through the fracture fragment. The pin plate is placed over the $\mathrm{K}$-wire and secured to the radius with a proximal screw. An additional $\mathrm{K}$-wire and additional proximal screws are placed to hold the reduction [23]. The pin plate has advantages in smaller fragments where screws may be too large to gain fixation (Fig. 5). 
Fig. 5 After plating the volar segment, dorsal-ulnar corner fixation was added to capture a large displaced articular segment, which helped secure the DRUJ

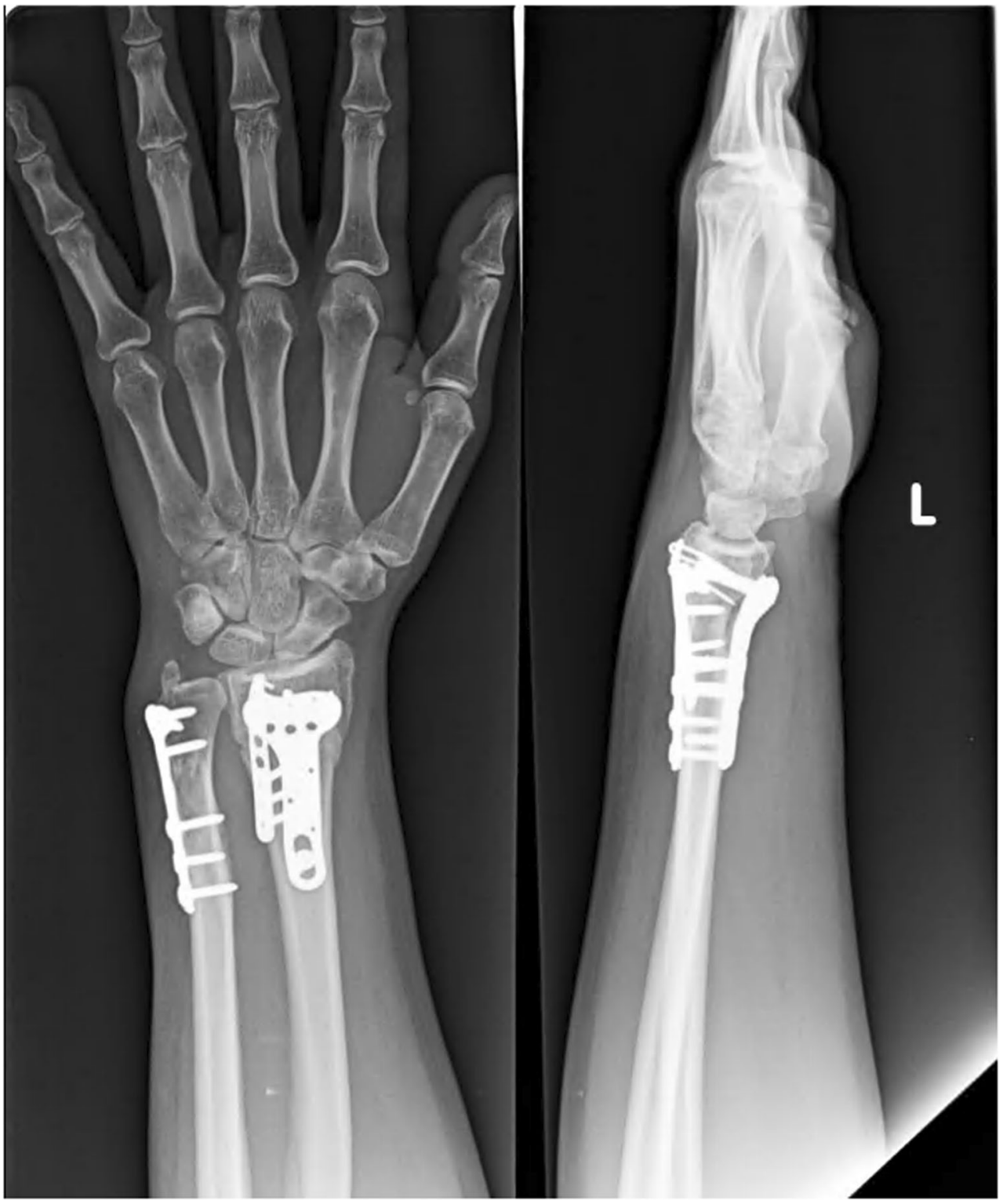

\section{Precontoured Plates}

A variety of dorsal precontoured plates exist, but usually are shaped in a "T," "L," or a teardrop morphology. Once the fracture is reduced and held with a K-wire, the plate can be secured to the radius proximally; then, screws can be placed to hold the fracture reduction.

\section{Outcomes}

Incongruity of the dorsal-ulnar corner of the distal radius can destabilize the distal radioulnar joint. When the forearm is in pronation, there will be an increase in volar-ulnar translation of the radius and the ulna head will become prominent dorsally [30]. Although early designs of dorsal plates were reported to have an unacceptable rate of extensor tendon rupture, modern designs with smoother edges and lower profiles have been shown to have a significantly reduced risk [31].

\section{Dorsal Wall}

\section{Anatomy}

The dorsal surface of the radius is irregular and the cortex is thin, leaving it at risk of fracture with dorsal angulation and comminution. The main irregularity is Lister's tubercle, which acts as a pulley for the extensor pollicis longus. The dorsal distal radius is concave and directly in contact with extensor tendons [21]. 


\section{Types of Fractures}

The dorsal wall can be involved in compression, bending, or high-energy fractures with comminution. Dorsal bending and axial loading injuries to the distal radius often result in comminution of the dorsal wall, with one or more dorsal wall fragments that may rotate into dorsiflexion. The presence of a dorsal wall fragment implies dorsal instability and can be associated with large metaphyseal defect [3]. Dorsal wall fractures also occur with dorsal shearing injuries.

\section{Approach}

It is a universal dorsal approach as described above.

\section{Types of Fractures}

Dorsal implants as described above would be desirable if large segments of articular surface are incongruent or if dorsal carpal displacement is present [32] (Fig. 6). Bone graft is also commonly placed behind the dorsal wall when required to support impacted fragments.

\section{Outcomes}

Kim et al. have shown that dorsal rim fractures appear larger on preoperative CT scans and, when displaced less than $2 \mathrm{~mm}$, can still be treated with volar plating [33].

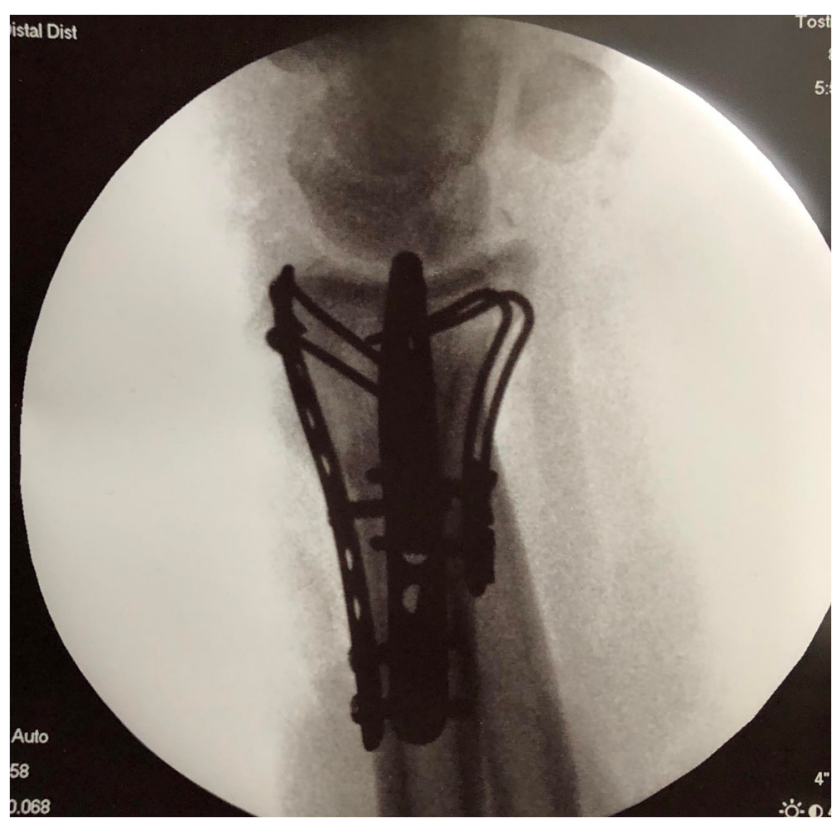

Fig. 6 A comminuted fracture requiring 3 plates in order to restore the articular congruity. The dorsal wall plate was required

\section{Impacted Intraarticular}

\section{Anatomy}

Impacted articular fractures can have several fragments involved. The fractures can be difficult to mobilize and reduce due to their small size and inaccessibility. These fractures can be visualized radiographically by noting subchondral bone in the metaphyseal area on AP or lateral views [3]. Additionally, a surgeon could use an arthroscope intraoperatively to assess congruity.

\section{Types of Fractures}

These fractures are typically higher energy, resulting in multiple fracture fragments in addition to the impacted articular pieces. A compression force generally drives the articular segment proximally.

\section{Approach}

Any of the above approaches can be used to correct impacted fragments. The rim or styloid fragments can be "booked open" or rotated on their ligamentous attachments to expose deeper segments. Those segments can be elevated with a tamp and usually assisted with bone graft for support.

\section{Types of Implants}

Any of the above implants can also be used to support an intraarticular impacted fragment. The surgeon should try to place the pins or screw within the subchondral bone of the fragment to create a "raft" for support.

\section{Outcomes}

Studies have shown that failure to restore a congruent joint surface leads to earlier onset posttraumatic arthrosis [34, 35].

\section{Conclusion}

Distal radius fractures are a very common injury seen by upper extremity surgeons. Fragment-specific fixation can be utilized in cases in which stabilization of specific articular segments is required. Radial styloid, volar rim, dorsal wall, dorsal-ulnar corner, and impacted intraarticular fragments may be secured with implants designed specifically for each individual fragment. Although these techniques can be technically demanding, they can be used independently or in combination with other fixation techniques to obtain accurate reductions and favorable functional outcomes. 


\section{Compliance with Ethical Standards}

Conflict of Interest Bryan A. Hozack and Rick J. Tosti each declare no potential conflicts of interest.

Human and Animal Rights and Informed Consent This article does not contain any studies with human or animal subjects performed by any of the authors.

Publisher's Note Springer Nature remains neutral with regard to jurisdictional claims in published maps and institutional affiliations.

\section{References}

Papers of particular interest, published recently, have been highlighted as:

- Of importance

-• Of major importance

1. Larsen CF, Lauritsen J. Epidemiology of acute wrist trauma. Int J Epidemiol. 1993;22(5):911-6.

2. Chung KC, Spilson SV. The frequency and epidemiology of hand and forearm fractures in the United States. J Hand Surg. 2001;26(5):90815.

3. Medoff RJ. Essential radiographic evaluation for distal radius fractures. Hand Clin. 2005;21(3):279-88.

4. Ilyas AM, Mudgal CS. Radiocarpal fracture-dislocations. J Am Acad Orthop Surg. 2008;16(11):647-55.

5. Berger RA, Landsmeer JM. The palmar radiocarpal ligaments: a study of adult and fetal human wrist joints. J Hand Surg. 1990;15(6):847-54.

6. Ritt MJ, Stuart PR, Berglund LJ, Linscheid RL, Cooney WP, An $\mathrm{KN}$. Rotational stability of the carpus relative to the forearm. J Hand Surg. 1995;20(2):305-11.

7. Robson AJ, See MS, Ellis H. Applied anatomy of the superficial branch of the radial nerve. Clin Anat. 2008;21(1):38-45.

8. Reichel LM, Bell BR, Michnick SM, Reitman CA. Radial styloid fractures. J Hand Surg. 2012;37(8):1726-41.

9. Lamas C, Llusà M, Méndez A, Proubasta I, Carrera A, Forcada P. Intraosseous vascularity of the distal radius: anatomy and clinical implications in distal radius fractures. Hand (N Y). 2009;4(4):418-23.

10. Koh S, Andersen CR, Buford WL, Patterson RM, Viegas SF. Anatomy of the distal brachioradialis and its potential relationship to distal radius fracture. J Hand Surg. 2006;31(1):2-8.

11. Melone CP. Articular fractures of the distal radius. Orthop Clin $\mathrm{N}$ Am. 1984;15(2):217-36.

12. Jupiter JB, Nunez F, Fricker R. Manual of fracture management: hand. New York: Thieme; 2016. (AO Trauma Series)

13. Fernandez DL, Geissler WB. Treatment of displaced articular fractures of the radius. J Hand Surg. 1991;16(3):375-84.

14. Ilyas AM. Surgical approaches to the distal radius. Hand (N Y). 2011;6(1):8-17.

15. Geissler WB, Clark SM. Fragment-specific fixation for fractures of the distal radius. J Wrist Surg. 2016;5(1):22-30 This article describes techniques for a fragment-specific fixation system with clinical photographs and imaging.

16. Mayfield JK, Johnson RP, Kilcoyne RK. Carpal dislocations: pathomechanics and progressive perilunar instability. J Hand Surg. 1980;5(3):226-41.
17.• Landgren M, Abramo A, Geijer M, Kopylov P, Tägil M. Fragmentspecific fixation versus volar locking plates in primarily nonreducible or secondarily redisplaced distal radius fractures: a randomized controlled study. J Hand Surg. 2017;42(3):156-165.e1 This article compares volar plates with fragment-specific fixation, showing equivalent outcomes, but emphasizing some of the potential complications of fragment-specific fixation.

18. Benson LS, Minihane KP, Stern LD, Eller E, Eshadri R. The outcome of intra-articular distal radius fractures treated with fragmentspecific fixation. J Hand Surg. 2006;31(8):1333-9.

19. Garner MR, Schottel PC, Thacher RR, Warner SJ, Lorich DG. Dual radial styloid and volar plating for unstable fractures of the distal radius. Am J Orthop (Belle Mead NJ). 2018;47(3).

20. Harness NG, Jupiter JB, Orbay JL, Raskin KB, Fernandez DL. Loss of fixation of the volar lunate facet fragment in fractures of the distal part of the radius. J Bone Joint Surg Am. 2004;86-A(9):1900-8.

21. Nana AD, Joshi A, Lichtman DM. Plating of the distal radius. J Am Acad Orthop Surg. 2005;13(3):159-71.

22. Kozin SH. Perilunate injuries: diagnosis and treatment. J Am Acad Orthop Surg. 1998;6(2):114-20.

23. Konrath GA, Bahler S. Open reduction and internal fixation of unstable distal radius fractures: results using the trimed fixation system. J Orthop Trauma. 2002;16(8):578-85.

24. Chin KR, Jupiter JB. Wire-loop fixation of volar displaced osteochondral fractures of the distal radius. J Hand Surg. 1999;24(3):525-33.

25.• O'Shaughnessy MA, Shin AY, Kakar S. Stabilization of volar ulnar rim fractures of the distal radius: current techniques and review of the literature. J Wrist Surg. 2016;5(2):113-9 This is a review of literature on volar-ulnar rim fracture treatment technique. The importance of reduction and fixation of this fragment is emphasized with helpful clinical photographs and radiologic studies.

26. Beck JD, Harness NG, Spencer HT. Volar plate fixation failure for volar shearing distal radius fractures with small lunate facet fragments. J Hand Surg. 2014;39(4):670-8.

27. Jupiter JB, Fernandez DL, Toh CL, Fellman T, Ring D. Operative treatment of volar intra-articular fractures of the distal end of the radius. J Bone Joint Surg Am. 1996;78(12):1817-28.

28. Jupiter JB. Complex articular fractures of the distal radius: classification and management. J Am Acad Orthop Surg. 1997;5(3):119-29.

29. Patterson RW, Van Niel M, Shimko P, Pace C, Seitz WH. Proprioception of the wrist following posterior interosseous sensory neurectomy. J Hand Surg. 2010;35(1):52-6.

30. Cole DW, Elsaidi GA, Kuzma KR, Kuzma GR, Smith BP, Ruch DS. Distal radioulnar joint instability in distal radius fractures: the role of sigmoid notch and triangular fibrocartilage complex revisited. Injury. 2006;37(3):252-8.

31. Spiteri M, Ng W, Matthews J, Power D, Brewster M. Three year review of dorsal plating for complex intra-articular fractures of the distal radius. J Hand Surg Asian Pac Vol. 2018;23(2):221-6.

32. Medoff RJ. Radiographic evaluation and classification of distal radius Fractures. In: Fractures and injuries of the distal radius and carpus. Elsevier; 2009 [cited 2018 Dec 22]. p. 17-31. Available from: https:// linkinghub.elsevier.com/retrieve/pii/B9781416040835000056

33. Kim JK, Yun Y-H, Kim DJ. The effect of displaced dorsal rim fragment in a distal radius fracture. J Wrist Surg. 2016;5(1):31-5.

34. Knirk JL, Jupiter JB. Intra-articular fractures of the distal end of the radius in young adults. J Bone Joint Surg Am. 1986;68(5):647-59.

35. Erhart S, Schmoelz W, Lutz M. Clinical and biomechanical investigation of an increased articular cavity depth after distal radius fractures: effect on range of motion, osteoarthrosis and loading patterns. Arch Orthop Trauma Surg. 2013;133(9):1249-55. 